\title{
Fabrication and analytical modeling of integrated heater and thermistor for antenna-coupled bolometers
}

\author{
Ajay Tiwari ${ }^{\mathrm{a}}$, Hiroaki Satoh ${ }^{\mathrm{a}}$, Makoto Aoki ${ }^{\mathrm{b}}$, Masanori Takeda ${ }^{\mathrm{b}}$, \\ Norihisa Hiromoto ${ }^{b}$, and Hiroshi Inokawa ${ }^{a, *}$ \\ ${ }^{a}$ Research Institute of Electronics, Shizuoka University, 3-5-1 Johoku, Naka-ku, Hamamatsu, 432-8011 Japan \\ ${ }^{\mathrm{b}}$ Graduate School of Engineering, Shizuoka University, 3-5-1 Johoku, Naka-ku, Hamamatsu, 432-8561 Japan
}

\begin{abstract}
Assuming the use in antenna-coupled bolometers to detect terahertz wave, integrated heater and thermistor were fabricated with titanium ( $\mathrm{Ti}$ ) on a silicon ( $\mathrm{Si}$ ) substrate, and its electrical responsivity was investigated at room temperature. To establish the dependence of microbolometer's responsivity on sizes and material characteristics of the thermistor and heater, an analytical model was made to describe the temperature rise with current in thermally isolated heater and resultant increase in electrical resistance. Although the behavior of the heater resistance could be reproduced by the model successfully, the responsivity coincides only for a bolometer of short heater length, suggesting the effect of slow response speed of the long heaters.
\end{abstract}

\section{Keywords: Microbolometer, Analytical Model, Responsivity, Titanium}

\section{Introduction}

Uncooled infrared (IR) focal plane arrays (FPAs) based on micromachined bolometer technology were successfully developed and found many applications in areas such as surveillance, firefighting, night vision, military, etc. [1],[2] The main advantage of microbolometer is their ability to operate at room temperature. Furthermore, uncooled bolometer devices have advantages such as lower cost, and better reliability without cooling system.

An ordinary IR microbolometer consists of absorber that receives electromagnetic radiation, and temperature sensitive resistor (thermistor). However, for longer wavelength, e.g. in the terahertz region, the absorber becomes too large to be structurally supported and thermally isolated, and then an antenna-coupled bolometer becomes more viable, in which the radiation is received by an antenna and converted to heat by a load resistor (heater). Till now, most of such microbolometers have been equipped with a thermistor which itself is a heater to absorb electromagnetic wave, but it is more advantageous in high sensitivity to optimize the thermistor and the heater independently by separating electrically but combining thermally each other [3].

*Corresponding author. TEL: +81-53-478-1308, FAX: +81-53-478-1651

Hiroshi Inokawa: Electronic mail:inokawa06@rie.shizuoka.ac.jp. 
Performance of such a microbolometer is mainly determined by (i) temperature coefficient of resistance (TCR) of the thermistor (ii) thermal isolation of the integrated heater and thermistor from the structural base, i.e. substrate. Since the temperature distribution in the heater is disturbed by the presence of the thermistor and additional leads, proper modeling is essential for evaluating and improving the characteristics of microbolometers [4]. This paper reports the fabrication and analytical modeling of the integrated heater and thermistor for antenna-coupled microbolometers.

\section{Fabrication Process}

Titanium (Ti) is selected as thermistor and heater materials because of its low thermal and electrical conductivities, immunity to electromigration, and prospective low flicker noise [4]-[6]. The process steps in the fabrication of microbolometer are as follows. The thermistor on thermally oxidized (TO) silicon ( $\mathrm{Si}$ ) substrate was formed by patterning of RF sputtered Ti thin film using photolithography as illustrated in Fig. 1(a). The interlayer $\mathrm{SiO}_{2}$ was deposited by electron cyclotron resonance (ECR) sputtering. The contact hole in ECR sputtered $\mathrm{SiO}_{2}$ was created by $\mathrm{CHF}_{3}$ reactive ion etching (RIE). After that, heater was fabricated by patterning of the sputtered Ti thin film as shown in Fig. 1(b). The deep-cavity for thermal isolation was formed by $\mathrm{CHF}_{3} \mathrm{RIE}$ and $\mathrm{SF}_{6}$ plasma etching with sacrificial Al mask as shown in Fig. 1(c). An optical micrograph of the top view of the fabricated microbolometer with their electrical connections is displayed in Fig. 1(d). The fabrication steps shown from Fig. 1(a) to (c) correspond to $\mathrm{YY}^{\prime}$ cross-section.

\section{Analytical modeling of fabricated microbolometer}

This section gives analytical expressions of temperature distribution along the heater line in the microbolometer and the resulting heater resistance as functions of heater current. The former is directly related to the bolometer output, and allows us to estimate the responsivity. The latter can be experimentally measured to extract the thermal resistance of the heater line.

\subsection{Temperature distribution}

Figure 2(a) shows the layout structure of the bolometer, in which voltage terminals are included for both heater and thermistor to assure accurate measurements. The temperature distribution and resistance of a one-dimensional suspended heater line have already been solved [7], and the solution is extended to include the heat loss caused by the leads of thermistor and the heater voltage terminals, based on the simplified model shown in Fig. 2(b). As it is obvious from Fig. 2(b), the heater line is divided in to two sections with lengths $L_{e}$ and $L . L_{e}$ represents the length between current terminal and voltage terminal in the heater line and $L$ is the heater length. Due to the symmetry of 
the bolometer structure, the analysis is carried out only for a half part of heater line. Here $T_{\mathrm{o}}$ is the ambient temperature, and $T_{\mathrm{v}}$ and $T_{\mathrm{c}}$ are the temperatures at voltage node and at central part of heater respectively. $C_{v}$ and $C_{c}$ are thermal conductances of voltage terminal leads of the heater and leads of the thermistor, respectively.

The differential equation describing the balance of the heat conduction loss and the Joule heating in an infinitesimal section of the heater line is given as [7],

$$
\frac{1}{r_{t}} \frac{d^{2} T}{d x^{2}}+I^{2} r_{e}\left[1+\alpha\left(T-T_{o}\right)\right]=0
$$

Where $x$ is length along heater length, $r_{\mathrm{t}}$ and $r_{\mathrm{e}}$ are the thermal resistance and electrical resistance per unit length of the heater at $T_{\mathrm{o}}$ respectively, and $\alpha$ is TCR of the heater. The $r_{\mathrm{e}}$ was determined from low-current DC I-V measurement while the $r_{\mathrm{t}}, C_{v}$ and $C_{c}$ were extracted from the $R$ versus $I^{2}$ curve which is discussed later. If the temperature $T$ is replaced with $\theta=1+\alpha\left(T-T_{\mathrm{o}}\right)$, Eq. (1) becomes,

$$
\frac{d^{2} \theta}{d x^{2}}+m^{2} \theta=0
$$

where $m^{2}=I^{2} \alpha r_{\mathrm{t}} r_{\mathrm{e}}$. The general solution to this is,

$$
\theta(x)=A \sin m x+B \cos m x .
$$

By extracting the constants $A, B, m$, using the first set of boundary conditions at the heater current terminal and the voltage node, and the second set at the voltage node and the central part of the heater, the temperature distribution along the heater line is expressed by equations, which have been derived in the Appendix A.

\subsection{Bolometer's electrical responsivity}

The bolometer's electrical responsivity is the measure of thermistor output $V_{\text {out }}$ with respect to input power applied to the heater. If a sinusoidal heater currents with a small amplitude $I$ is assumed, average input power $P_{\text {in }}$ (average) $=P_{\text {in }}($ peak $) / 2=I^{2} R_{\mathrm{o}} / 2$, where $R_{\mathrm{o}}=r_{\mathrm{e}} L$ is the heater resistance at $T_{\mathrm{o}}$, and the root-mean-square output voltage of the bolometer is $V_{\text {out }}(\mathrm{RMS})=V_{\text {out }}($ peak-to-peak $) /(2 \sqrt{2})$. The bolometer responsivity $R_{\mathrm{b}}=V_{\text {out }}(\mathrm{RMS}) / P_{\text {in }}$ (average) is derived in Appendix A as shown in Eq. (A.13) and expanded as follows,

$$
R_{b}=\frac{I_{t h} R_{t h} \alpha_{t h}}{2 \sqrt{2}} \frac{C_{v} r_{t} L_{e} L+L+4 L_{e}+4 L_{e}^{2} / L}{C_{v} r_{t} L_{e} L C_{c}+4 C_{v} L_{e}+L C_{c}+4+2 L_{e} C_{c}}
$$

Here, $I_{\mathrm{th}}, R_{\mathrm{th}}$ and $\alpha_{\mathrm{th}}$ are bias current, resistance and TCR of the thermistor, respectively. Inputting the measured $R_{\mathrm{th}}$ 
and $\alpha_{\mathrm{th}}$, extracted $r_{\mathrm{t}}, C_{v}$ and $C_{c}$, designed values of $L$ and $L_{\mathrm{e}}$, and set value of $I_{\mathrm{th}}$, electrical responsivity can be estimated.

\subsection{Heater resistance}

Heater resistance as a function of current provides information on thermal resistances if TCR is known. In order to formulate electrical resistance of the heater between the two voltage terminals, the average temperature $\theta_{\mathrm{av}}$ in section 2 of Fig. 2(b) have to be calculated, and the heater resistance is then given by $R=R_{o} \theta_{\mathrm{av}}$. Expanding the $\theta_{\mathrm{av}}$ in the Taylor series with respect to $m$ and taking the first two terms as mentioned in the Appendix A by Eq. (A.15), we obtain

$$
R \approx R_{o}\left[1+\frac{1}{48} \frac{\left(\begin{array}{l}
L^{3} C_{c} r_{t}+C_{v} r_{t}^{2} L_{e} L^{3} C_{c}+16 C_{v} r_{t} L_{e} L^{2}+8 L_{e} C_{c} r_{t} L^{2} \\
+16 L^{2}+12 L L_{e}^{2} C_{c} r_{t}+96 L L_{e}+96 L_{e}^{2}
\end{array}\right)}{2 L_{e} C_{c} r_{t}+L C_{c} r_{t}+4 C_{v} r_{t} L_{e}+C_{v} r_{t}^{2} L_{e} L C_{c}+4} \alpha r_{e} r_{t} I^{2}\right]
$$

The slope of the $R-I^{2}$ characteristics is expressed by the second term of Eq. (5). Based on the slopes for devices with different $L$ 's and the $r_{\mathrm{e}}$ value obtained from $R_{\mathrm{o}}$ 's for the same, parameters $r_{\mathrm{t}}, C_{\mathrm{c}}$ and $C_{\mathrm{v}}$ can be extracted by solving simultaneous equations or minimizing error between experimental and theoretical slopes expressed by Eq.

\section{Results and Discussion}

After the device fabrication, the basic parameters were measured, calculated and listed in Table 1. Heater length $L$ is varied from 200 to $800 \mu \mathrm{m}$, but the $L_{\mathrm{e}}$ is fixed at $35 \mu \mathrm{m}$. Thickness of the heater/thermistor was measured by stylus profilometer, and width and lengths were designed values. Resistance and TCR were obtained by DC I-V measurement at $240-300 \mathrm{~K}$, and the values for $300 \mathrm{~K}$ are listed. The similar TCR values were also reported by different groups [8],[9].

Thermal resistance of the heater line and the supporting $\mathrm{SiO}_{2}$ was evaluated based on the phenomenon that the resistance of the heater increases with increasing DC current. The increase of the resistance as a function of square of the current is shown in Fig. 3. The resistance linearly increases with the square of the current. The amount of the resistance change increases with heater length. This tendency for isolated wire is also observed and discussed by Zhang et al. [7]. We have the extended electro-thermal analytical solution reported by Zhang et al. to incorporate 
heat loss caused by the leads of thermistor and the heater voltage terminal as described in Section 3 and Appendix A. It can be seen from Fig. 3 that the experimental data (symbols) fit very well with the analytical model (solid line) for each length. Fitting parameters for the analytical model are $r_{\mathrm{t}}, C_{\mathrm{c}}, C_{\mathrm{v}}$ which are $1.97 \times 10^{10} \mathrm{~K} /(\mathrm{Wm}), 3.20 \times 10^{-7} \mathrm{~W} / \mathrm{K}$, and $2.38 \times 10^{-6} \mathrm{~W} / \mathrm{K}$, respectively. The $r_{\mathrm{t}}$ coincides well with $1.79 \times 10^{10} \mathrm{~K} /(\mathrm{Wm})$ calculated from the reported values of thermal conductances $1.32 \mathrm{~W} /(\mathrm{mK})$ for $\mathrm{SiO}_{2}[10]$ and $21.9 \mathrm{~W} /(\mathrm{mK})$ for $\mathrm{Ti}$ [5]. In order to compare the thermal conductance of the present device with the reported value, the conductance $G_{\text {total }}$ between the central part of the heater $\left(T_{\mathrm{c}}\right)$ and the ambience $\left(T_{\mathrm{o}}\right)$ can calculated by the following equation.

$$
G_{\text {total }}=C_{c}+2\left[L \frac{r_{t}}{2}+\left\{C_{v}+\left(L_{e} r_{t}\right)^{-1}\right\}^{-1}\right]^{-1}
$$

$G_{\text {total }}$ decreases monotonically as the $L$ increases from $1.22 \times 10^{-6} \mathrm{~W} / \mathrm{K}$ at $200 \mu \mathrm{m}$ to $5.66 \times 10^{-7} \mathrm{~W} / \mathrm{K}$ at $800 \mu \mathrm{m}$.

These $G_{\text {total }}$ values are one to two orders of magnitude higher than those of infrared focal plane arrays [11], but the direct comparison may not be appropriate due to the differences in device sizes and types (i.e. antenna-coupled type and absorber type). Additional discussion on the relationship among thermal conductance, capacitance and cutoff frequency will be made in the later section.

Furthermore, the analytical model was also used to calculate temperature distribution in each heater. The temperature profile for the input power of $10 \mu \mathrm{W}$ is plotted against position in Fig. 4. At the both ends of the heater, the temperature is $300 \mathrm{~K}$, and increases gradually towards the center position. However, at the center there is a drop in the temperature, which is due to the heat loss through the thermistor leads. There are also deflection points near the ends of the heater caused by the heat loss by the voltage terminal leads of the heater.

The electrical responsivity of the fabricated microbolometers is measured by applying AC electrical power up to $50 \mu \mathrm{W}$ at $10 \mathrm{~Hz}$. Thermistor output was detected by lock-in amplifier under the bias current of $0.5 \mathrm{~mA}$. Thermistor output voltage with respect to the heater input power is shown in Fig. 5. The responsivity i.e. the slope of the line has a maximum value $14.6 \mathrm{~V} / \mathrm{W}$ for heater length $L$ of $200 \mu \mathrm{m}$ and monotonically decreases as the $L$ increases. The responsivity expected from the analytical model is $18.4 \mathrm{~V} / \mathrm{W}$ for the $L$ of $200 \mu \mathrm{m}$, but increases as the $L$ increases as shown in Fig. 6.

This discrepancy is presumably caused by the lower cutoff frequency of the bolometer response for longer $L$ relative to the measurement frequency of $10 \mathrm{~Hz}$. Since the analytical model can only deal with the DC (static) cases, we supplementarily use the electro-thermal circuit simulation [12] to analyze dynamic behavior including frequency response. The simulation is based on the duality of electrical and thermal circuits, i.e. electrical current, voltage, 
resistance and capacitance correspond to heat flow, temperature, thermal resistance and capacitance, respectively. Then, the electrical and thermal circuits can be simulated simultaneously by an ordinary circuit simulator such as SPICE [13] even if there are interactions between these two circuits, e.g. Joule heat flows into the thermal circuit and temperature change affects the electrical resistance via TCR. At a low frequency $(0.1 \mathrm{~Hz})$, as illustrated in Fig. 6 , the simulated result coincides well with the analytical model, and, at a high frequency $(10 \mathrm{~Hz})$, the simulation successfully reproduces the reverse behavior of the experimental result, i.e. reduction of the responsivity with $L$.

In order to get a better understanding, the frequency responses for microbolometers with different $L$ 's are simulated and plotted in Fig. 7. It can be seen that, at a low frequency $(0.1 \mathrm{~Hz})$, the responsivity increases with $L$, but the tendency is reversed at a high frequency $(10 \mathrm{~Hz})$ due to the reduction of the cutoff frequency $f_{\mathrm{c}}$. We extracted $f_{\mathrm{c}}$ from the simulated result, and then calculated the effective thermal capacitance $C_{\text {eff }}$ by

$$
C_{\text {eff }}=G_{\text {total }} / 2 \pi f_{c}
$$

where $G_{\text {total }}$ is the thermal conductance given by Eq. (6). Note that the $C_{\text {eff }}$ is an effective value because the heat generation is distributed over the entire heater and is not concentrated at the central part of the heater.

In Fig. $8, G_{\text {total }}, C_{\text {eff }}$ and $f_{\mathrm{c}}$ are plotted with respect to $L$. It is found that the $f_{\mathrm{c}}$ decreases more rapidly than the $G_{\text {total }}$ does while the latter is inversely proportional to the responsivity at low frequencies, and therefore the reverse behavior, i.e. reduction of the responsivity with $L$, is observed.

Please note that, although the electro-thermal simulation covers static and dynamic operations, and seems more versatile, the proposed analytical model is still useful because the device usually operates at a lower frequency than $f_{\mathrm{c}}$, and the simpler calculation involved in the model makes the parameter $\left(r_{\mathrm{t}}, C_{\mathrm{c}}, C_{\mathrm{v}}\right.$, etc.) extraction faster and performance estimation more straightforward.

This report focuses on the analysis and modeling of the integrated heater and thermistor, and the overall design of the antenna-coupled bolometer is reported elsewhere [14],[15]. However, it is worthwhile to mention the antenna structure that may affect the future design of the heater and thermistor. If we assume half-wave dipole antenna operating at $1 \mathrm{THz}$, the resonant antenna length and load (heater) resistance become $52 \mu \mathrm{m}$ and $38 \Omega$, respectively, for substrate $(\mathrm{Si})$ dielectric constant of 11.67 and antenna width-length ratio of 0.1 [16]. In this case, length of the heater placed at the center of antenna should be sufficiently shorter than the antenna length and practically around 10 $\mu \mathrm{m}$. This size reduction would eliminate the issue of low $f_{\mathrm{c}}$ in the present device, but negatively affect the responsivity as suggested by Figs. 6-8. Since the target heater resistance is fixed and therefore the thermal conductance cannot be independently reduced due to the correlation between electrical and thermal conductances, the 
responsivity must be maintained or improved by increasing $R_{\mathrm{th}}$, and reducing $C_{\mathrm{c}}$ and $C_{\mathrm{v}}$ as indicated by Eq. (4). This will be accomplished by making the thermistor and leads narrower and longer based on the advanced lithography. The proposed analytical model would be useful in designing and performance estimation of such a bolometer.

\section{Conclusion}

A series of integrated heater and thermistor was fabricated and its operation was analyzed. The change in the heater resistance with respect to the current was evaluated based on the newly developed analytical model, and thermal resistances in the structure were extracted. With these values, temperature distribution along the heater line and the electrical responsivity were estimated. The experimental responsivity is close to the analytical one only for short heater length. As opposed to the expectation, bolometer with longer heater showed smaller responsivity, due to the lower cutoff frequency than the measurement frequency. The developed analytical model is useful in analyzing and optimizing the antenna-coupled bolometers especially when there are thermistor and other leads stemming from the heater line.

\section{Acknowledgement}

This study is partially supported by Industry-Academia Collaborative R \& D from Japan Science and Technology Agency, JST.

\section{APPENDIX A}

\section{Temperature distribution}

Assuming the temperature $T_{\mathrm{c}}\left(\theta_{\mathrm{c}}\right)$ at the central part of the bolometer, and $T_{\mathrm{v}}\left(\theta_{\mathrm{v}}\right)$ at the voltage node of the heater,

Eq. (3) can be solved in section 1 of Fig. 2(b) with boundary conditions,

$$
\begin{aligned}
& \theta\left(x_{1}=0\right)=B_{1}=1 \\
& \theta\left(x_{1}=L_{e}\right)=A_{1} \sin m L_{e}+B_{1} \cos m L_{e}=\theta_{v}
\end{aligned}
$$

to obtain,

$$
A_{1}=\frac{\theta_{v}-\cos m L_{e}}{\sin m L_{e}} .
$$

In section 2, boundary conditions are, 


$$
\begin{aligned}
& \theta\left(x_{2}=0\right)=B_{2}=\theta_{v} \\
& \theta\left(x_{2}=L / 2\right)=A_{2} \sin m L / 2+B_{2} \cos m L / 2=\theta_{c}
\end{aligned}
$$

resulting in,

$$
A_{2}=\frac{\theta_{c}-\theta_{v} \cos m L / 2}{\sin m L / 2}
$$

Solutions for sections 3 and 4 can be obtained by considering the symmetry of the structure.

For the balance of heat flowing into and out of the voltage node of the heater, obtained is,

$$
-\left.\frac{1}{r_{t}} \frac{d T}{d x_{1}}\right|_{x 1=L e}+\left.\frac{1}{r_{t}} \frac{d T}{d x_{2}}\right|_{x 2=0}=C_{v}\left(T_{v}-T_{o}\right)
$$

where $C_{\mathrm{v}}$ is the thermal conductance of the voltage terminal lead. Eq. (A.5) can be reduced to,

$$
\frac{m}{r_{t}}\left(\frac{1-\theta_{v} \cos m L_{e}}{\sin m L_{e}}+\frac{\theta_{c}-\theta_{v} \cos m L / 2}{\sin m L / 2}\right)=C_{v}\left(\theta_{v}-1\right)
$$

For the balance of heat flowing into and out of the central node of the bolometer, obtained is,

$$
-\left.\frac{2}{r_{t}} \frac{d T}{d x_{2}}\right|_{x 2=L / 2}=C_{c}\left(T_{c}-T_{o}\right)
$$

where $C_{\mathrm{c}}$ is the thermal conductance of the thermistor leads. Eq. (A.7) can be reduced to,

$$
\frac{2 m}{r_{t}} \frac{\theta_{v}-\theta_{c} \cos m L / 2}{\sin m L / 2}=C_{c}\left(\theta_{c}-1\right)
$$

By solving the Eqs. (A.6) and (A.8) simultaneously, $\theta_{\mathrm{c}}$ and $\theta_{\mathrm{v}}$ are obtained as follows.

$$
\theta_{c}=\frac{-\left[\begin{array}{l}
C_{v} r_{t}^{2} \sin \left(m L_{e}\right) \sin (m L / 2) C_{c}+m \sin (m L / 2) \cos \left(m L_{e}\right) C_{c} r_{t} \\
+m \sin \left(m L_{e}\right) \cos (m L / 2) C_{c} r_{t}+2 C_{v} r_{t} \sin \left(m L_{e}\right) m+2 m^{2}
\end{array}\right]}{\left[\begin{array}{l}
-C_{v} r_{t}^{2} \sin \left(m L_{e}\right) \sin (m L / 2) C_{c}-2 C_{v} r_{t} \sin \left(m L_{e}\right) m \cos (m L / 2) \\
-m \sin (m L / 2) \cos \left(m L_{e}\right) C_{c} r_{t}-2 m^{2} \cos \left(m L_{e}\right) \cos (m L / 2) \\
-m \sin \left(m L_{e}\right) \cos (m L / 2) C_{c} r_{t}+2 m^{2} \sin \left(m L_{e}\right) \sin (m L / 2)
\end{array}\right]}
$$




$$
\theta_{v}=\frac{-\left[\begin{array}{l}
C_{v} r_{t}^{2} \sin \left(m L_{e}\right) \sin (m L / 2) C_{c}+m \sin (m L / 2) C_{c} r_{t} \\
+m \sin \left(m L_{e}\right) C_{c} r_{t}+2 C_{v} r_{t} \sin \left(m L_{e}\right) m \cos (m L / 2)+2 m^{2} \cos (m L / 2)
\end{array}\right]}{\left[\begin{array}{l}
-C_{v} r_{t}^{2} \sin \left(m L_{e}\right) \sin (m L / 2) C_{c}-2 C_{v} r_{t} \sin \left(m L_{e}\right) m \cos (m L / 2) \\
-m \sin (m L / 2) \cos \left(m L_{e}\right) C_{c} r_{t}-2 m^{2} \cos \left(m L_{e}\right) \cos (m L / 2) \\
-m \sin \left(m L_{e}\right) \cos (m L / 2) C_{c} r_{t}+2 m^{2} \sin \left(m L_{e}\right) \sin (m L / 2)
\end{array}\right]}
$$

Once $\theta_{\mathrm{c}}$ and $\theta_{\mathrm{v}}$ are obtained, coefficients $A_{1}, B_{1}, A_{2}$ and $B_{2}$ are given by Eqs. (A.2), (A.1), (A.4) and (A.3), respectively, and finally the temperature distribution along the heater line can be calculated by Eq. (3) and $T=T_{\mathrm{o}}+(\theta-1) / \alpha$.

\section{Bolometer responsivity}

The bolometer output $V_{\text {out }}$ reflects the increase in the thermistor resistance caused by the rise of the temperature $\theta_{\mathrm{c}}$ at the central part of the bolometer. In the first order approximation, the temperature rise is proportional to the input power $P_{\text {in }}$ to the heater. This proportionality can be seen in the primary part of the Taylor series of $\theta_{\mathrm{c}}$ expanded with respect to $m$.

$$
\left.\theta_{c} \approx \theta_{c}\right|_{m=0}+\left.\frac{d \theta_{c}}{d m}\right|_{m=0} m+\left.\frac{1}{2} \frac{d^{2} \theta_{c}}{d m^{2}}\right|_{m=0} m^{2}=1+\left.\frac{1}{2} \frac{d^{2} \theta_{c}}{d m^{2}}\right|_{m=0} \frac{\alpha r_{t}}{L} p_{i n}
$$

$P_{\text {in }}$ is given by $I^{2} R_{\mathrm{o}}$ for small heater current $I$, where $R_{\mathrm{o}}=r_{\mathrm{e}} L$ is the heater resistance at the ambient temperature. Then the $V_{\text {out }}$ is expressed by

$$
V_{\text {out }}=I_{t h} R_{t h} \alpha_{t h}\left(T_{c}-T_{o}\right)=\left.I_{t h} R_{t h} \alpha_{t h} \frac{\theta_{C}-1}{\alpha} \approx I_{t h} R_{t h} \alpha_{t h} \frac{d^{2} \theta_{c}}{d m^{2}}\right|_{m=0} \frac{r_{t}}{2 L} p_{\text {in }},
$$

where $I_{\mathrm{th}}, R_{\mathrm{th}}$ and $\alpha_{\mathrm{th}}$ are thermistor bias current, resistance and TCR of thermistor, respectively. If a sinusoidal heater current with an amplitude $I$ is assumed, average input power is $P_{\text {in }}$ (average) $=P_{\text {in }} / 2$, and the root-mean-square output voltage of the bolometer is $V_{\text {out }}(\mathrm{RMS})=V_{\text {out }} /(2 \sqrt{ } 2)$. The bolometer responsivity $R_{\mathrm{b}}=V_{\text {out }}(\mathrm{RMS}) / P_{\text {in }}($ average $)$ is then given by,

$$
R_{b}=\left.\frac{I_{t h} R_{t h} \alpha_{t h} r_{t}}{2 \sqrt{2} L} \frac{d^{2} \theta_{c}}{d m^{2}}\right|_{m=0}
$$




\section{Heater resistance}

In order to find the resistance of the heater between the two voltage terminals, average temperature $\theta_{\mathrm{av}}$ in section 2 of Fig. 2(b) (equal to the one in section 3) have to be calculated.

$$
\begin{aligned}
& \theta_{a v}= \frac{2}{L} \int_{0}^{L / 2}\left(A_{2} \sin m x_{2}+B_{2} \cos m x_{2}\right) d x_{2}=\frac{2}{L m}\left[A_{2}(1-\cos m L / 2)+B_{2} \sin m L / 2\right] \\
&-2\left[\begin{array}{l}
-2 C_{v} r_{t}^{2} \sin \left(m L_{e}\right) \sin (m L / 2) C_{c}-m \sin (m L / 2) C_{c} r_{t} \\
+2 m^{2} \cos ^{2}(m L / 2)+2 \cos (m L / 2) C_{v} r_{t}^{2} \sin \left(m L_{e}\right) \sin (m L / 2) C_{c} \\
m \sin (m L / 2) \cos \left(m L_{e}\right) C_{c} r_{t} \cos (m L / 2)-2 m^{2} \\
-m \sin \left(m L_{e}\right) C_{c} r_{t}-2 C_{v} r_{t} \sin \left(m L_{e}\right) m \\
-m \sin (m L / 2) \cos \left(m L_{e}\right) C_{c} r_{t}+\cos (m L / 2) m \sin (m L / 2) C_{c} r_{t} \\
+2 C_{v} r_{t} \sin \left(m L_{e}\right) m \cos ^{2}(m L / 2)+m \sin \left(m L_{e}\right) C_{c} r_{t} \cos ^{2}(m L / 2)
\end{array}\right] \\
& m L\left[\begin{array}{l}
C_{v} r_{t}^{2} \sin \left(m L_{e}\right) C_{c}-\cos ^{2}(m L / 2) C_{v} r_{t}^{2} \sin \left(m L_{e}\right) C_{c} \\
+2 m \sin (m L / 2) C_{v} r_{t} \sin \left(m L_{e}\right) \cos (m L / 2)+m \cos \left(m L_{e}\right) C_{c} r_{t} \\
-m \cos \left(m L_{e}\right) C_{c} r_{t} \cos ^{2}(m L / 2)+2 m^{2} \sin (m L / 2) \cos \left(m L_{e}\right) \cos (m L / 2) \\
+m \sin (m L / 2) \sin \left(m L_{e}\right) \cos (m L / 2) C_{c} r_{t}-2 m^{2} \sin \left(m L_{e}\right) \\
+2 m^{2} \sin \left(m L_{e}\right) \cos ^{2}(m L / 2)
\end{array}\right]
\end{aligned}
$$

Then the heater resistance is given by $R=R_{o} \theta_{\mathrm{av}}$. In the first order approximation, temperature rise and resistance increase are proportional to the input power to the heater or the square of the heater current. This proportionality can be seen in the primary part of the Taylor series of $\theta_{\mathrm{av}}$ expanded with respect to $m$.

$$
R \approx R_{o}\left(1+\left.\frac{d \theta_{a v}}{d m}\right|_{m=0} m+\left.\frac{1}{2} \frac{d^{2} \theta_{a v}}{d m^{2}}\right|_{m=0} m^{2}\right)=R_{o}\left(1+\left.\frac{1}{2} \frac{d^{2} \theta_{a v}}{d m^{2}}\right|_{m=0} \alpha r_{e} r_{t} I^{2}\right)
$$




\section{References}

[1] P. W. Kruse, Uncooled IR focal plane arrays, Proc. SPIE 2552 (1995) 556-563 .

[2] S. Chen, H. Ma, S. Xiang, X. Yi, Fabrication and performance of microbolometer arrays based on nanostructured vanadium oxide thin films, Smart Mater. Struct. 16 (2007) 696-700.

[3] N. Hiromoto, Terahertz detectors and sensing for the ground use, in: 9th Takayanagi Kenjiro Meml. Symposium \& 4th International Symposium on Nanovision Science, Hamamatsu, Japan, October 29-30, 2007.

[4] A. Tanaka, S. Matsumoto, N. Tsukamoto, S. Itoh, K. Chiba, T. Endoh, A. Nakazato, K. Okuyama, Y. Kumazawa, M. Hijikawa, H. Gotoh, T. Tanaka, N. Teranishi, Infrared focal plane array incorporating silicon IC process compatible bolometer, IEEE Trans. Electron Devices 43 (1996) 1844-1850.

[5] The Wikimedia Foundation, Inc., http://en.wikipedia.org/wiki/Titanium.

[6] Y. L. Cheng, B. J. Wei, F. H. Shih, Y. L. Wang, Stability and Reliability of Ti/TiN as a Thin Film Resistor, ECS J. Solid State Science and Technology 2 (2013) Q12-Q15.

[7] S. Zhang, Y. Yang, S. M. Sadeghipour, M. Asheghi, Thermal characterization of the $144 \mathrm{~nm}$ GMR layer using microfabricated suspended structure, in: Proc. ASME Summer Heat Transfer Conf., Las Vegas, Nevada, USA, July 21-23, 2003, HT2003-47270.

[8] W.-B. Song, J. J. Talghader, Design and characterization of adaptive microbolometers, J. Micromech. Microeng. 16 (2006) 1073-1079.

[9] R. K. Bhan, R. S. Saxena, C. R. Jalwania, S. K. Lomash, Uncooled Infrared Microbolometer Arrays and their Characterisation Techniques, Defense Science J. 59 (2009) 580-589.

[10] R. Kato and I. Hatta, Thermal Conductivity and Interfacial Thermal Resistance: Measurements of Thermally Oxidized $\mathrm{SiO}_{2}$ Films on a Silicon Wafer Using a Thermo-Reflectance Technique, Int. J. Thermophys. 29 (2008) 2062-2071.

[11] M. Kimata, M. Ueno, M. Takeda, Toshiki Seto, SOI diode uncooled infrared focal plane arrays, Proc. of SPIE, 6127 (2006) 61270X-1 61270X-11.

[12] A. Tiwari, H. Satoh, M. Aoki, M. Takeda, N. Hiromoto, H. Inokawa, Analysis of Microbolometer Characteristics for Antenna-Coupled Terahertz Detectors, Asian J. Chem. 25 (2013) S358-S360. 
[13] Linear Technology Corporation, LTspice IV, http://www.linear.com/designtools/software.

[14] M. Aoki, M. Takeda, N. Hiromoto, Electromagnetic Simulation for THz Antenna-Coupled Microbolometers Operated at Room Temperature, Makara J. of Technology 17 (2013) 1-6.

[15] N. Hiromoto, A. Tiwari, M. Aoki, H. Satoh, M. Takeda, H. Inokawa, Room-Temperature THz Antenna-Coupled Microbolometer with a Joule-Heating Resistor at the Center of a Half-Wave Antenna, in: 39th Int. Conf. on Infrared Millimeter and Terahertz Waves (IRMMW-THz), Tucson, AZ, USA, September 14-19, 2014, to be presented.

[16] M. Kominami, D. M. Pozar, D. H. Schaubert, Dipole and slot elements and arrays on semi-infinite substrate, IEEE Trans. on Antenna and Propagation, AP-33 (1985) 600-607. 


\section{Biographies}

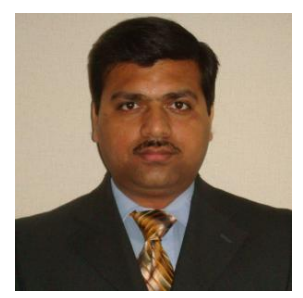

Ajay Tiwari received his Ph.D. degree at Department of Physics, Indian Institute of Technology, Bombay (IIT Bombay), Mumbai, INDIA, in 2007, where he was engaged in fabrication and characterization of magnetic granular thin films. He has also worked in magnetic nanoparticles, rare-earth doped GaN films. He joined Nanosystem Integration Laboratory in 2012, where he is involved in fabricating THz detector using Electron Beam Lithography. He has been trying to analyze the observed responsivities, NEP using electro-thermal circuit simulation.

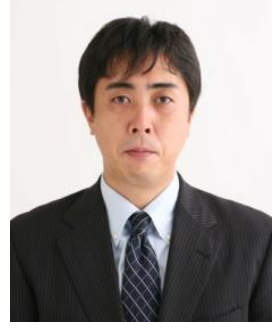

Hiroaki Satoh received the B.E. degree from the Muroran Institute of Technology, Hokkaido, Japan, in 1999, and the M.E. and the Ph.D. degrees from Hokkaido University, Hokkaido, in 2001 and 2004, respectively. He was a Research Associate with the University of Tokushima, Tokushima, Japan, from 2004 to 2007. Since 2007, he has been an Assistant Professor with the Research Institute of Electronics, Shizuoka University, Hamamatsu, Japan. His current research interests include the computational electromagnetics, and its application to silicon nanodevices for advanced photonics. Dr. Satoh is a member of the Japan Society of Applied Physics, the Institute of Electronics, Information and Communication Engineers of Japan, and the Institute of Electrical Engineers of Japan.

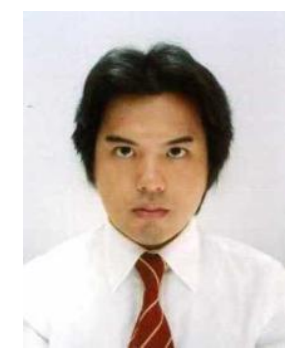

Makoto Aoki received B.S., M.E., and D.E. degrees from Shizuoka University in 2007, 2009, and 2013, respectively. $\mathrm{He}$ is currently a postdoctoral researcher of Graduate School of Engineering at Shizuoka University and engaged on a study of antenna design of terahertz antenna-coupled microbolometer. His research interests include terahertz passive imaging and sensing. 


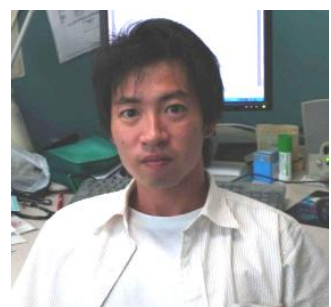

Masanori Takeda was born in Hiroshima, Japan, on January 28, 1973. He received the B.S. and M.S. degrees in electronics from Yamanashi University, Kofu, Japan, in 1995 and 1997, respectively, and the Ph.D. degree in radio astronomy from the Graduate University for Advanced Studies, Kanagawa, Japan, in 2002. From 2001 to 2009, he was with the National Institute of Technology, where he was engaged in the development of heterodyne receivers with $\mathrm{NbN}$ SIS junctions in the terahertz band. From April to November in 2009, he was with the National Astronomical Observatory of Japan, where he was engaged in the development of the Atacama Large Millimeter/submillimeter Array (ALMA) band-10 receivers. He is currently a Lecturer of Graduate School of Engineering, Shizuoka University, Hamamatsu, Japan. His research interests include superconducting devices and terahertz technologies.

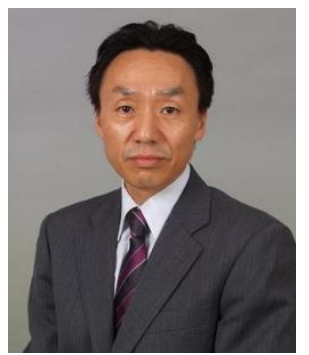

Norihisa Hiromoto received B.S. and Ph.D. degrees in Physics from Kyoto University in 1978 and 1985. His professional activities since joining Communications Research Laboratory (CRL) in 1984 have included research on detector and laser technologies in terahertz region, an asbestos fiber monitor using optical technique. He was the director of Kansai Advanced Research Center of CRL in 2001 and moved to the Ministry of Internal Affairs and Communications (MIC) as a Senior Planning Officer in 2003. He is presently a professor of Shizuoka University after 2005. He has authored and co-authored more than 60 scientific and technical papers and holds four patents in terahertz an optical technology.

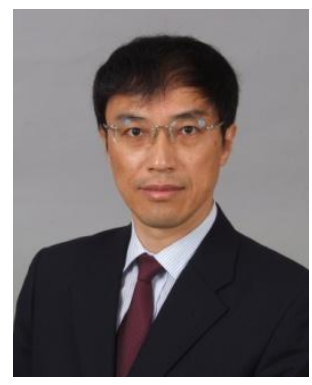

Hiroshi Inokawa received the Ph.D. degree in electrical engineering from Kyoto University, Kyoto, Japan, in 1985. In the same year, he joined the Atsugi Electrical Communications Laboratories, Nippon Telegraph and Telephone Corporation (NTT), Kanagawa, Japan. Since then, he has been engaged in research and development of scaled-down CMOS devices and silicon single-electron devices. In 2006, he became a professor of the Research Institute of Electronics, Shizuoka University, Hamamatsu, Japan, where he has been studying nanodevices for advanced circuits and systems.

Prof. Inokawa is a member of the Institute of Electrical and Electronics Engineers, the Japan Society of Applied Physics, the Institute of Electronics, Information and Communication Engineers of Japan, and the Institute of Electrical Engineers of Japan. 


\section{Figure with figure captions}

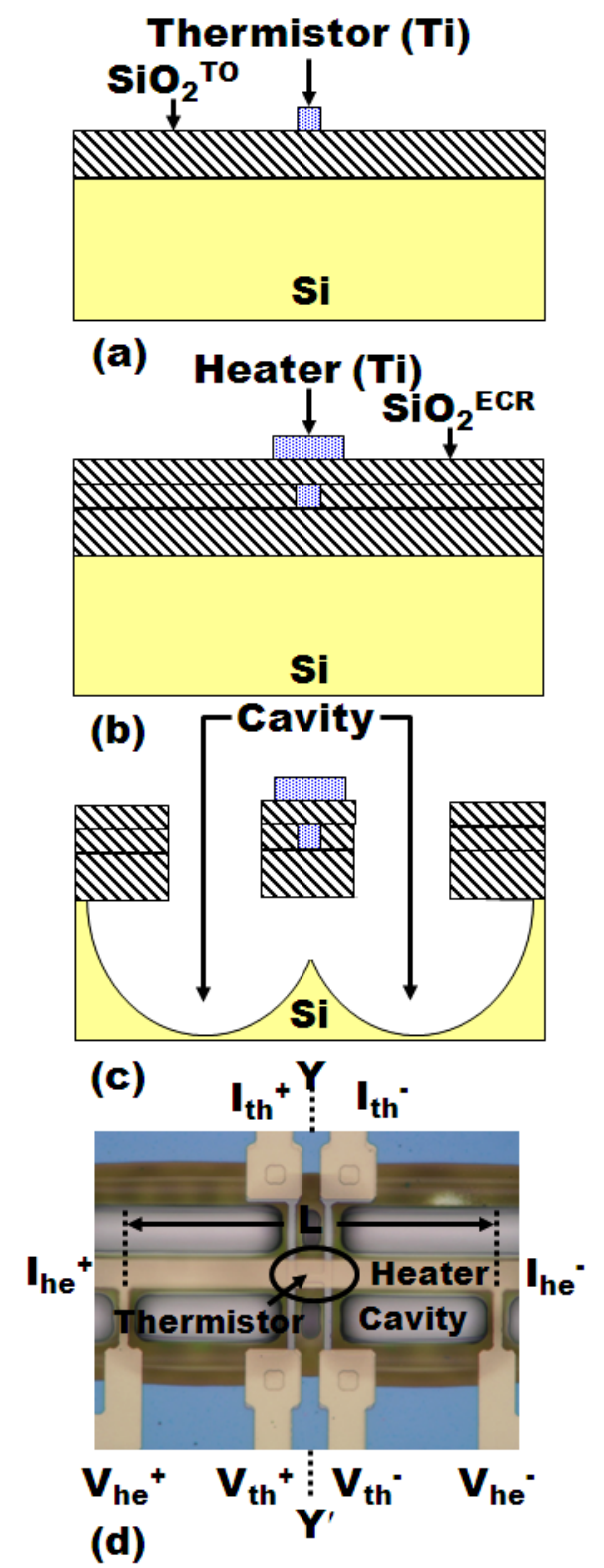

Fig. 1. Ti microbolometer fabrication steps along $Y^{\prime}{ }^{\prime}$ cross-section (a) patterned thermistor, (b) heater pattern

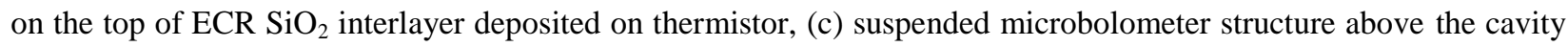
in Si substrate, and (d) optical micrograph of the top view of device with individual layer details and their electrical connections. $I_{h e}$ and $I_{t h}$ are current terminals for heater and thermistor, respectively, and $V_{\text {he }}$ and $V_{\text {th }}$ are voltage terminals for the same. 


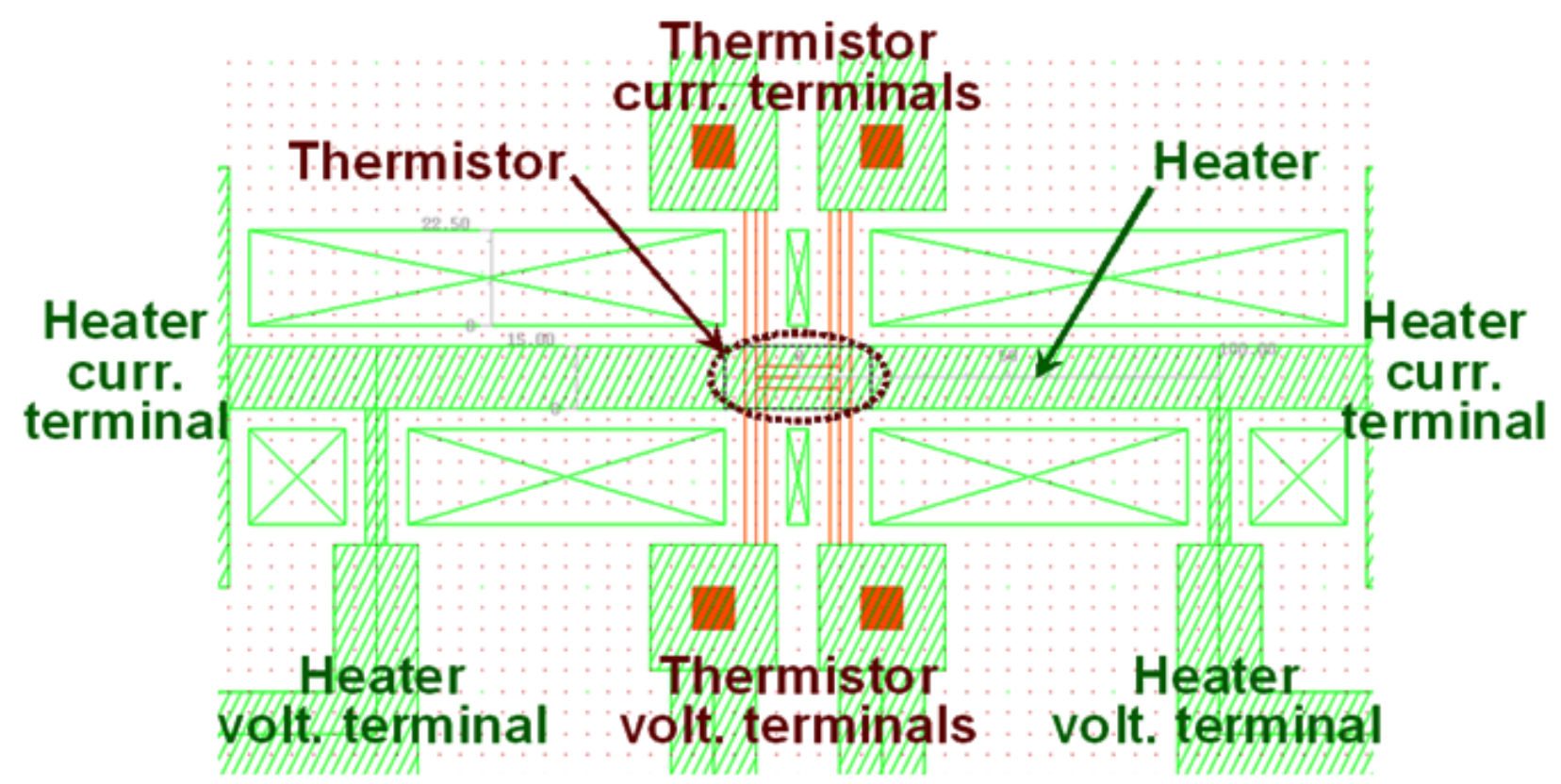

(a)
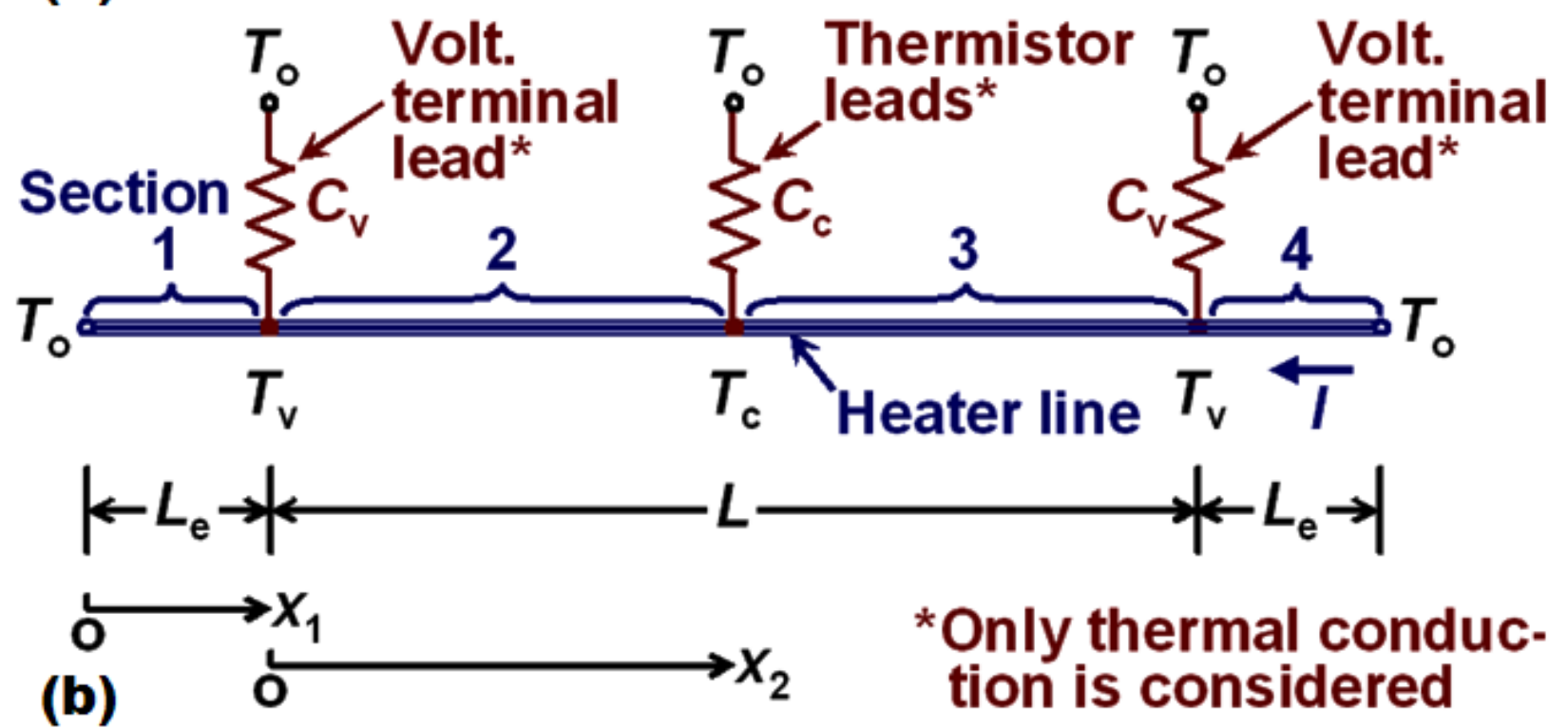

Fig. 2. (a) Schematic structure of microbolometer, and (b) simplified electro-thermal model of microbolometer. $C_{\mathrm{v}}$, and $C_{\mathrm{c}}$ are thermal conductance of voltage terminal and thermistor leads, respectively. $\mathrm{T}_{0}, \mathrm{~T}_{\mathrm{v}}$ and $\mathrm{T}_{\mathrm{c}}$ are the temperatures at the respective points.

\section{Ajay Tiwari $\boldsymbol{e t ~ a l . ~}$}




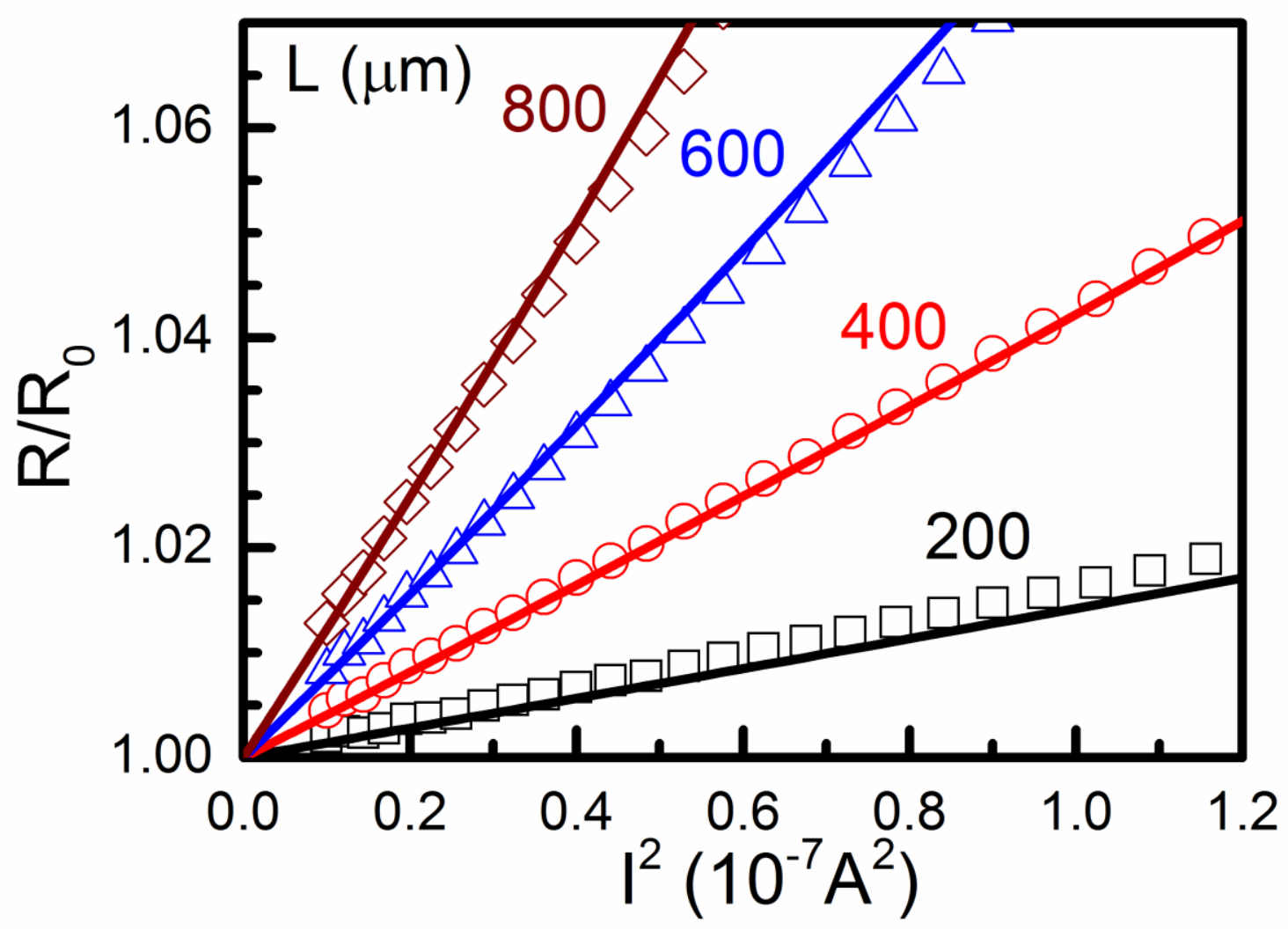

Fig. 3. Normalized heater resistance of the Ti microbolometers as a function of square of the applied current for each heater with different length. The symbols show experimental data whereas solid lines show those by the analytical model.

\section{Ajay Tiwari et al.}




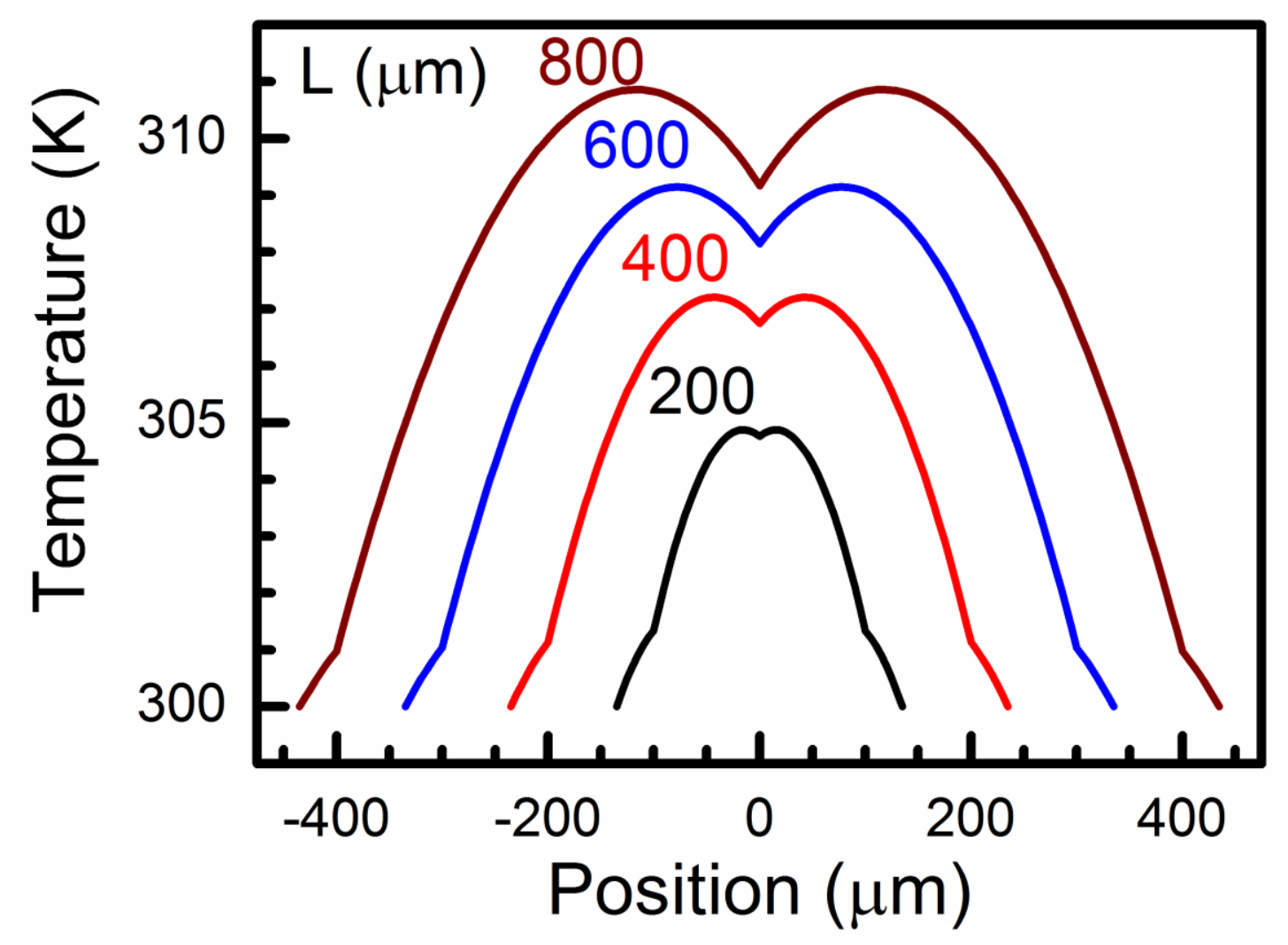

Fig. 4. Temperature distribution along the heater length for each heater predicted by the analytical model. The heater input power is $10 \mu \mathrm{W}$.

\section{Ajay Tiwari et al.}




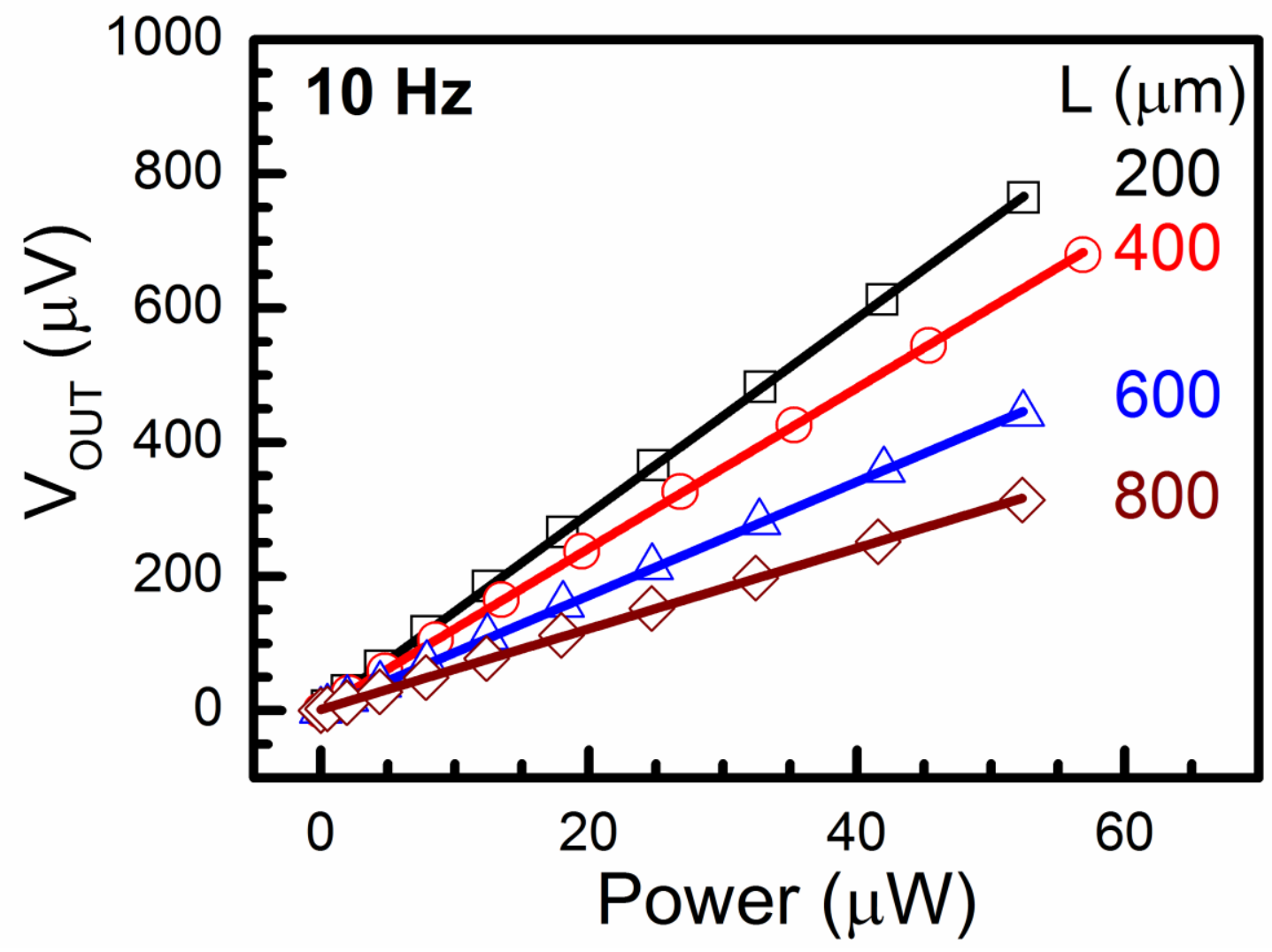

Fig. 5. Experimental thermistor output voltage versus heater input power for different heater lengths. The thermistor bias current is $0.5 \mathrm{~mA}$.

\section{Ajay Tiwari et al.}




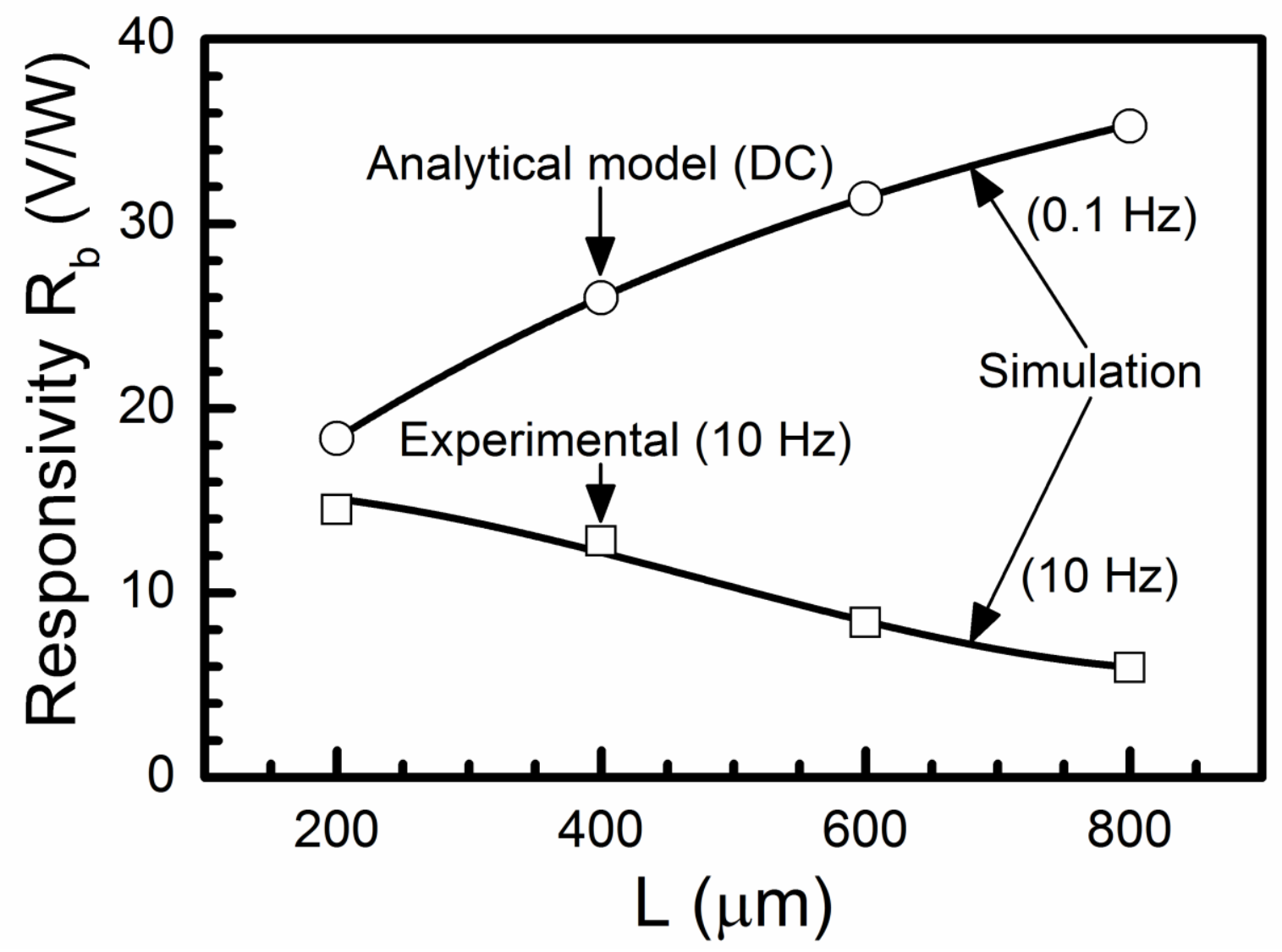

Fig. 6. The experimental and analytical responsivities of Ti microbolometer as functions of heater length. The square symbols show the experimental result while circular symbols show analytical result, solid lines show simulated results at different frequencies.

\section{Ajay Tiwari et al.}




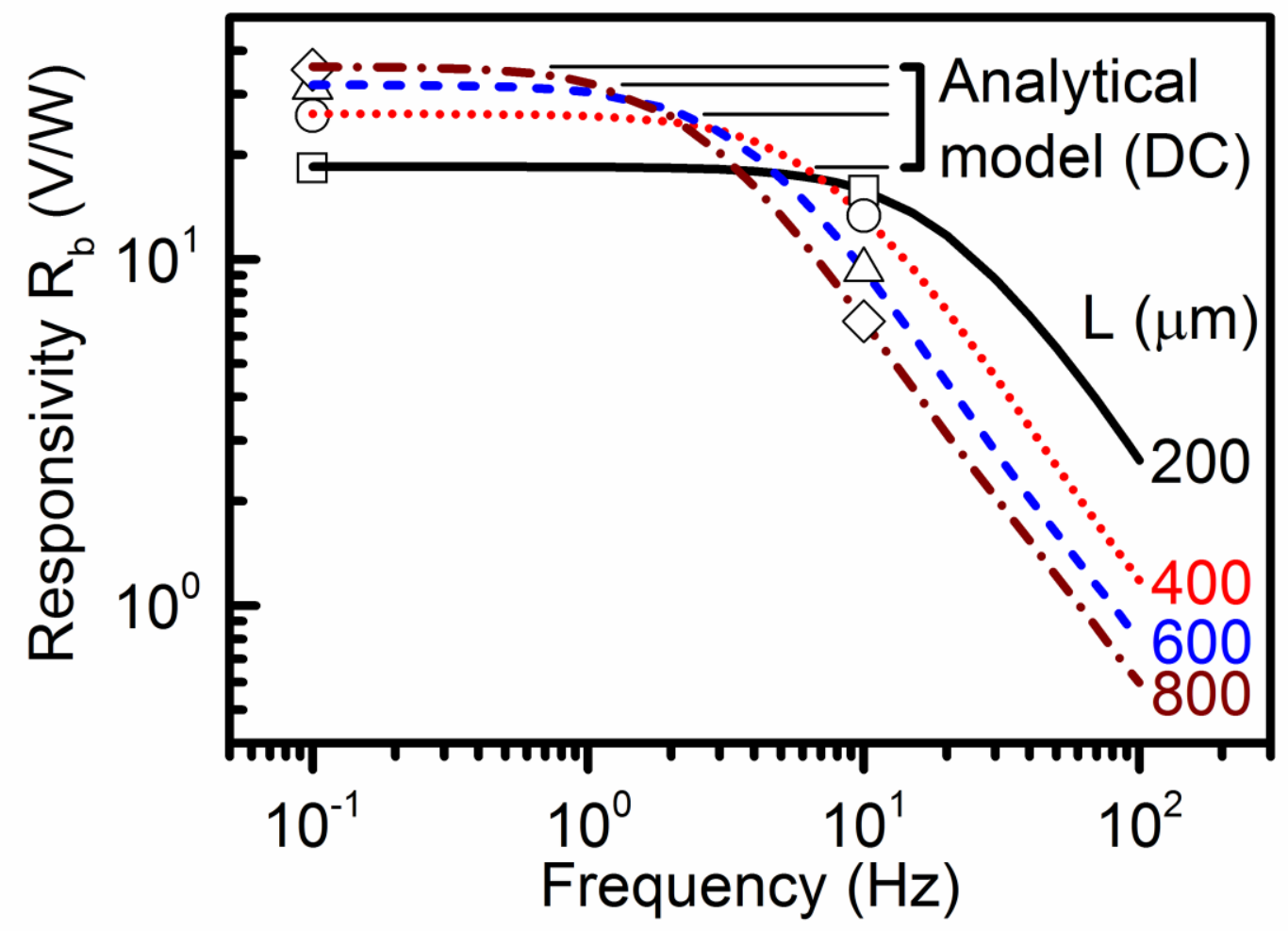

Fig. 7. Simulated frequency response curve for each heater. The symbols show the responsivity of each heater at 0.1 and $10 \mathrm{~Hz}$. Results from the analytical model are also shown as horizontal lines for comparison.

\section{Ajay Tiwari et al.}




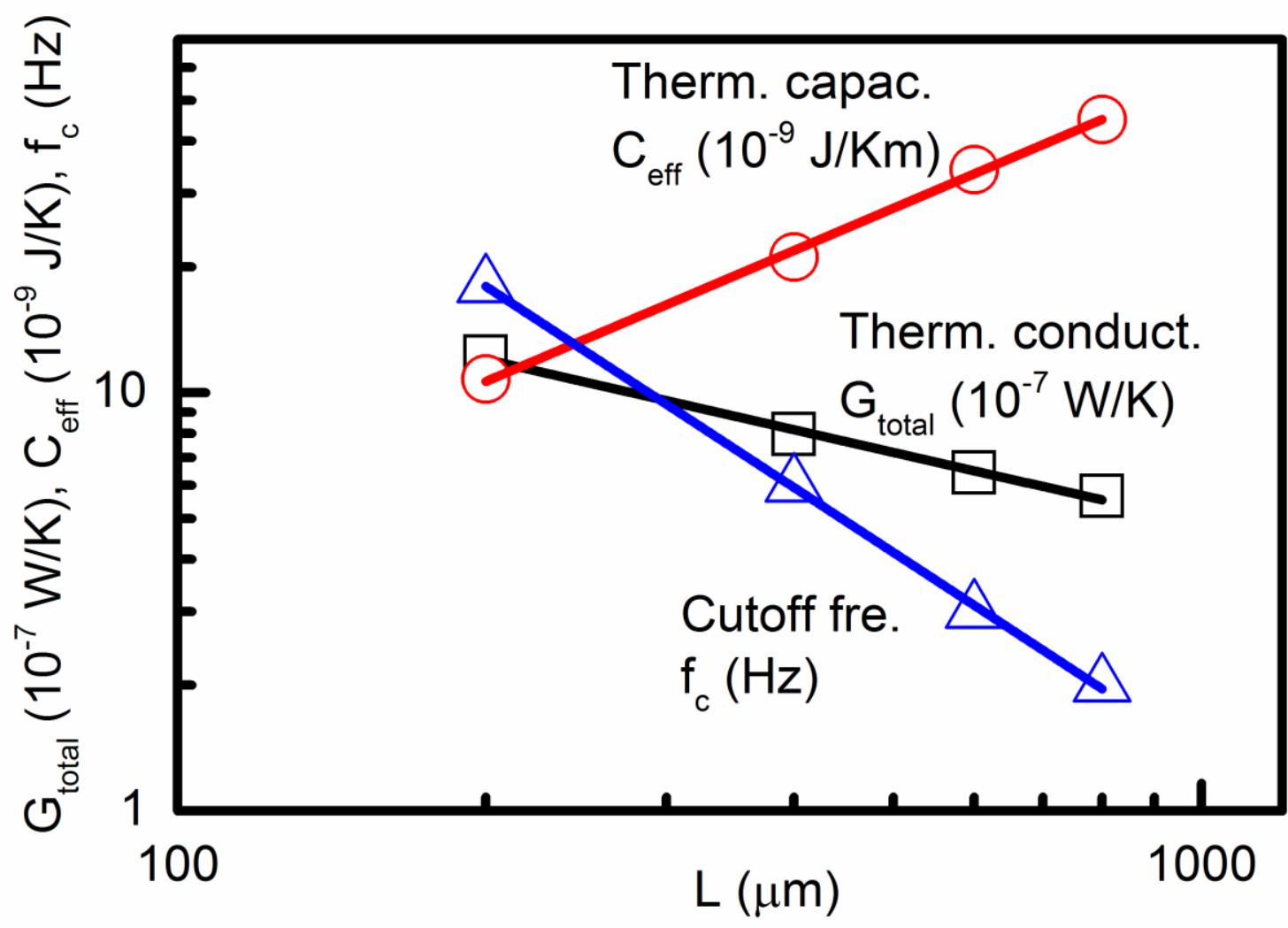

Fig. 8. Thermal conductance, thermal capacitance and cutoff frequency as a function of heater length.

Ajay Tiwari et al. 


\section{Table and captions}

Table 1. Device dimensions and resistance parameters

\begin{tabular}{|c|c|c|}
\hline Parameters & Heater & Thermistor \\
\hline Thickness (nm) & 118 & 47 \\
\hline Width $(\mu \mathrm{m})$ & 15 & 5 \\
\hline Length $(\mu \mathrm{m})$ & $200,400,600,800$ & 20 \\
\hline Resistance & $0.846 \Omega / \mu \mathrm{m}$ & $44.0 \Omega$ \\
\hline TCR $\left(\mathrm{K}^{-1}\right)$ & $2.14 \times 10^{-3}$ & $2.48 \times 10^{-3}$ \\
\hline $\mathrm{SiO}^{\mathrm{TO}}$ thickness (nm) & 424 & \\
\hline $\mathrm{SiO}_{2}{ }^{\mathrm{ECR}}$ thickness (nm) & 89 & \\
\hline Cavity depth $(\mu \mathrm{m})$ & 41 & \\
\hline
\end{tabular}

